\title{
Ataxia and Parkinsonism in Metronidazole Neurotoxicity
}

\author{
Ryan T. Muir(D), Marck Mercado, Victoria R. Walter, Nikhita Singhal(D, \\ Priscilla Kwan, Walter Kucharczyk, Nathan M. Stall, Peter L. Carlen
}

Keywords: Metronidazole, Toxic encephalopathy, Parkinsonism, Magnetic resonance imaging doi:10.1017/cjn.2020.156

Can J Neurol Sci. 2021; 48: 273-274

A 41-year-old man with Crohn's disease presented with progressive gait instability, slowness of movement, dysarthria, and lower extremity numbness which developed over 2 weeks. He did not experience focal or progressive weakness, diplopia, dysphagia, visual changes, or cognitive decline. His medications included a daily multivitamin, both ciprofloxacin and metronidazole for 8 years for infected perianal ulcers, and ustekinumab a monoclonal antibody directed against interleukin-12 and -23 - which he started 1 year prior to his current presentation.

On examination, he had hypophonia, bradykinesia, dysdiadochokinesia, and gait ataxia. There was no evidence of appendicular ataxia or resting tremor. His cranial nerve examination was unremarkable, tone was unremarkable, strength was full, and deep tendon reflexes were $2+$ and symmetric with the exception of absent Achilles tendon reflexes bilaterally. Plantar responses were flexor bilaterally. He had a symmetric length-dependent sensory neuropathy with loss of vibration and pin-prick in the distal lower extremities. Gait was wide based and bradykinetic. Blood work including a complete blood count, thyroid-stimulating hormone, vitamin B12 level, electrolytes, liver enzymes, and liver synthetic function tests were all within normal limits. An MRI brain revealed symmetric T2-fluid attenuation inversion recovery (FLAIR) hyperintense lesions involving the bilateral dentate nuclei and superior olivary nuclei in the dorsal pons (Figure 1A) and bilateral posterior putamen (Figure 1B). This pattern is concerning for a metabolic or toxic encephalopathy, but is typical of metronidazoleinduced encephalopathy (MIE). ${ }^{1-3}$ While dentate and superior olivary nuclei involvement is classic, putamen involvement is infrequently described in MIE. ${ }^{2}$ Other regions that can be affected in MIE include the midbrain (tectum, red nucleus, and periaqueductal gray matter (PAG)), dorsal pons (affecting the vestibular and abducens nuclei), dorsal medulla, corpus callosum, and white matter of cerebral hemispheres. ${ }^{2}$ Cessation of metronidazole resulted in improved bradykinesia and ataxia over 1 week, with complete resolution of symptoms 3 weeks afterward stopping the drug. He remains symptom free to this day.

While our patient's imaging was typical for MIE, other diagnoses were entertained. Although our patient did not consume alcohol, he did undergo total colonic resection 17 years prior, so nutritional deficiencies were considered. Wernicke's encephalopathy (WE) is an important differential diagnosis as similar regions can be affected as in MIE. ${ }^{4}$ However, in WE, T2-hyperintense lesions typically affect the mammillary bodies, medial thalamus, floors of the third and fourth ventricles, PAG, and midbrain tectum. $^{2}$ The exact mechanism of MIE remains uncertain. In addition to a cerebellar syndrome, MIE can result in optic neuropathy, encephalopathy, seizures, and autonomic and peripheral neuropathies. ${ }^{5}$ Our case is a unique contribution in that bilateral putamen involvement could have mediated this patient's mild Parkinsonism, characterized by bradykinesia and hypophonia.

A repeat MRI brain performed 6 months after cessation of metronidazole revealed complete resolution of symmetric T2FLAIR hyperintense lesions involving the bilateral dentate nuclei and superior olivary nuclei in the dorsal pons and bilateral putamen (see Figure 2). However, unexpectedly, there was an evolution of subtle diffuse T2-FLAIR hyperintensity within the splenium of the corpus callosum and the adjacent white matter of the parietal lobes - in keeping with a toxic or metabolic abnormality. Interestingly, the patient did not endorse any evolution of confusion, headaches, impaired memory, difficulties with speed of information processing, language dysfunction, apraxia, or seizures. He had not started any new medications, denies use of recreational drugs or alcohol, or progressive weight loss or nutritional deficiency. The patient does not have hypertension. While the resolution of his previous dentate nuclei, superior olivary nuclei, and bilateral putamen lesions is in keeping with resolving metronidazole toxicity, it is unknown whether the interval evolution of the subtle splenial and parietal white matter T2-FLAIR signal changes could be (i) a delayed manifestation of prior metronidazole toxicity that was not visualized at the first MRI or (ii) potentially secondary to ustekinumab. A retrospective case series does support that there can be delayed or incomplete resolution of splenial lesions in metronidazole toxicity. ${ }^{2}$ Furthermore, reversible posterior leukoencephalopathy syndrome has been described in the context of ustekinumab previously. ${ }^{6}$

From the Adult Neurology Residency Training Program, Department of Medicine, Division of Neurology, University of Toronto, Toronto, Ontario, Canada (RTM, PK); Undergraduate Medical Education, University of Toronto, Toronto, Ontario, Canada (MM); Internal Medicine Residency Training Program, Department of Medicine, University of Toronto, Toronto, Ontario, Canada (VRW); Psychiatry Residency Training Program, Department of Psychiatry, University of Toronto, Toronto, Ontario, Canada (NS); Joint Department of Medical Imaging, University of Toronto, Toronto, Ontario, Canada (WK); Division of General Internal Medicine and Geriatrics, Sinai Health System, University of Toronto, Toronto, Ontario, Canada (NMS); and Department of Medicine, Division of Neurology, University Health Network, University of Toronto, Toronto, Ontario, Canada (PLC)

Received March 29, 2020. Final Revisions Submitted June 23, 2020. Date of AcCeptance July 11, 2020.

Correspondence to: Dr. Peter L. Carlen, Toronto Western Hospital, 399 Bathurst St. 5W-442, Toronto, ON M5T 2S8, Canada. Email: carlen@uhnresearch.ca 

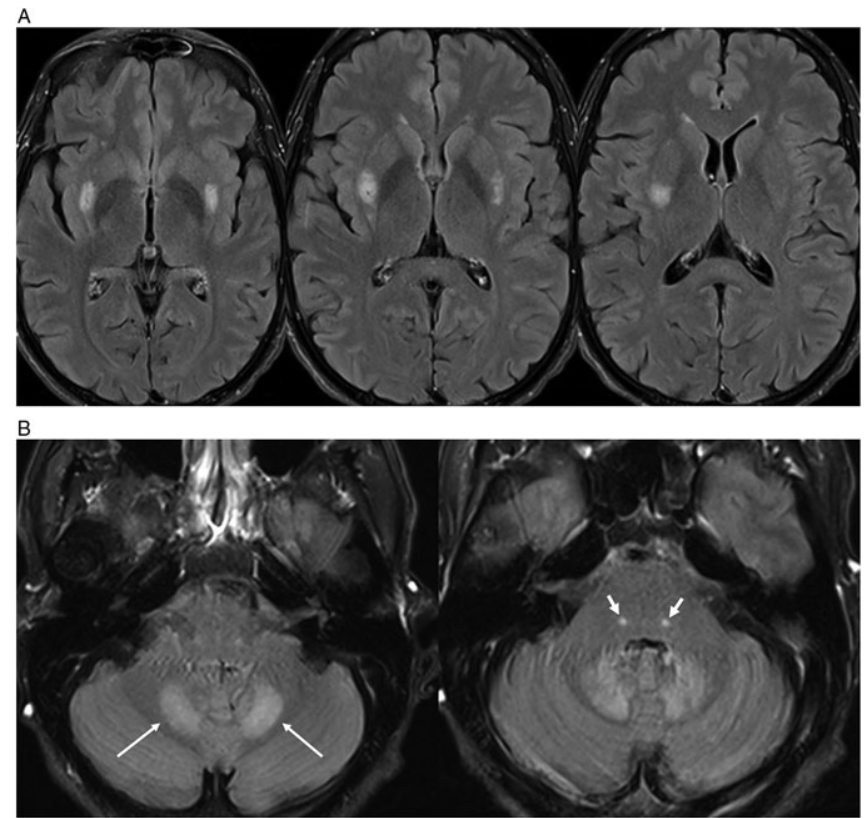

Figure 1: 1.5T MRI brain T2-FLAIR axial images highlighting hyperintense lesions in the (A) posterior aspect of the putamen bilaterally and (B) the dentate nuclei of the cerebellum (thin white arrow) as well as focal tegmental lesions of the superior olivary nuclei of the dorsal pons bilaterally (thick white arrow). These were not associated with diffusion restriction on diffusion weighted imaging, nor any susceptibility on susceptibility weighted imaging or enhancement on post-gadolinium T1-weighted imaging.
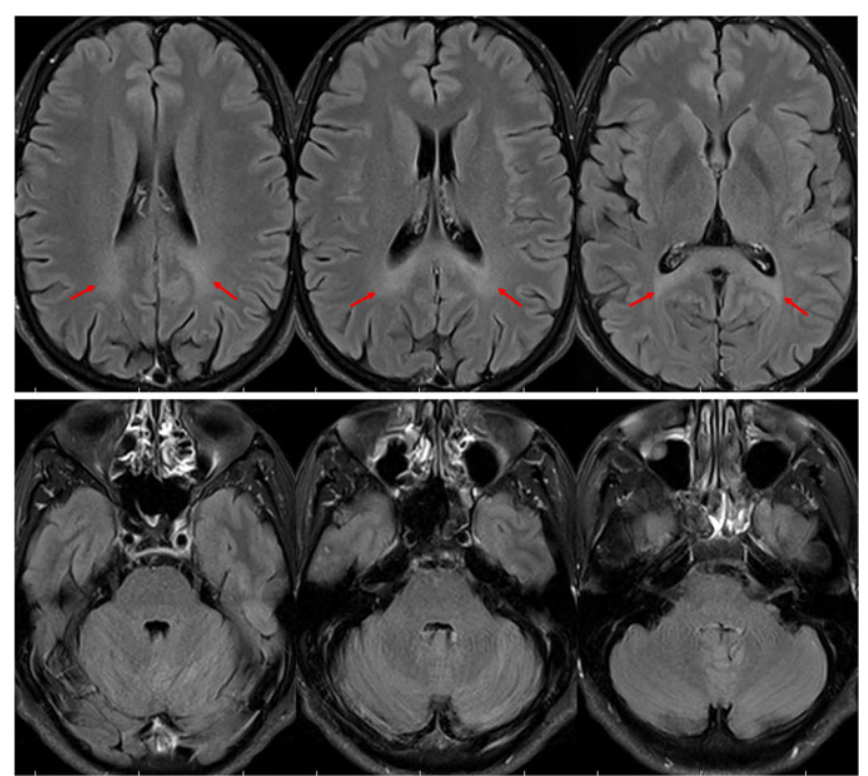

Figure 2: $3 T$ MRI brain T2-FLAIR axial images highlighting resolution of previous hyperintense lesions in the posterior aspect of the putamen bilaterally, dentate nuclei of the cerebellum, as well as the superior olivary nuclei bilaterally. There is interval evolution of subtle diffuse T2FLAIR hyperintensity within the splenium of the corpus callosum and the adjacent white matter of the parietal lobes (red arrows). These were not associated with diffusion restriction on diffusion weighted imaging, nor any susceptibility on susceptibility weighted imaging or enhancement on post-gadolinium T1-weighted imaging.
Reversible corpus callosum lesions have been described with anti-TNF therapies such as adalimumab. ${ }^{7}$

In conclusion, while the clinical spectrum of metronidazole central nervous system neurotoxicity is fairly broad and typically presents with ataxia and associated bilateral dentate nuclei T2FLAIR lesions on MRI, there are fewer reports of bilateral putamen involvement. This case portrays both typical and unique clinical and neuroradiographic manifestations of MIE. To the best of our knowledge, this is the only report of reversible Parkinsonism in the context of metronidazole neurotoxicity. Furthermore, this report also raises the possibility of delayed neuroradiographic manifestations of metronidazole toxicity as, at the time of the initial MRI, T2-FLAIR lesions were not noted in the splenium of corpus callosum and deep white matter of the parietal lobes. In the absence of other identifiable metabolic perturbations or toxicities, the evolution of these manifestations on subsequent MRI could represent delayed onset; however, it could also be related to ustekinumab therapy as it has been rarely described in the literature previously. Recognizing the clinical spectrum of and neuroimaging hallmarks of MIE is paramount as the cessation of metronidazole typically results in reversibility and obviates unnecessary investigations.

\section{Disclosures}

The authors do not have any conflicts of interest to disclose relevant to this publication.

\section{Statement of Authorship}

RTM: report conception and design, manuscript preparation, and image preparation. MM, VRW, NS, and PK: manuscript preparation and image preparation. PLC and NMS: manuscript preparation, acquisition of data, and final approval of manuscript.

\section{Consent}

The patient provided their informed and written consent to publish this case.

\section{REFERENCES}

1. Woordruff BK, Wijdicks EFM, Marshall WF. Reversible metronidazole-induced lesions of the cerebellar dentate nuclei. N Engl J Med. 2002;346:68-9.

2. Kim E, Na DG, Kim EY, Kim JH, Son KR, Chang KH. MR imaging of metronidazole-induced encephalopathy: Lesion distribution and diffusion-weighted imaging findings. Am J Neuroradiol. 2007;28:1652-8.

3. Jiménez MT, Gándara PS, Espay AJ. Teaching NeuroImages: The dentate sign in subacute cerebellar ataxia: Metronidazole neurotoxicity. Neurology. 2020;94:e878-e879.

4. Lefkowitz A, Shadowitz S. Reversible cerebellar neurotoxicity induced by metronidazole. CMAJ. 2018;190:E961.

5. Sarna JR, Furtado S, Brownell AKW. Neurologic complications of metronidazole. Can J Neurol Sci. 2013;40:768-76.

6. Gratton D, Szapary P, Goyal K, Fakharzadeh S, Germain V, Saltiel P. Reversible posterior leukoencephalopathy syndrome in a patient treated with ustekinumab. Arch Dermatol. 2011;147: 1197-202.

7. Park JW, Seok HY, Kim Y, Kim BJ. Reversible corpus callosal lesions associated with the use of adalimumab for ulcerative colitis. J Clin Neurol. 2017;13:209-11. 